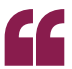

...CarD is

an essential

component of

the stringent

response in

mycobacteria.

$\Rightarrow$ BACTERIAL PHYSIOLOGY

\title{
Another CarD in the pack
}

Mycobacterium tuberculosis establishes a persistent infection that can last for the lifetime of the host. To do so, this intracellular pathogen uses a variety of transcriptional control mechanisms, including the stringent response, to withstand host stresses. In a new paper that was recently published in Cell, Christina Stallings and colleagues now reveal that CarD is an essential component of the stringent response in mycobacteria.

Stallings et al. were initially interested in characterizing the mycobacterial response to double-stranded DNA breaks. They looked at the transcriptional response of Mycobacterium smegmatis to the endonuclease I-SceI and found that one of the most highly upregulated genes was the uncharacterized gene carD. Further investigations revealed that carD expression is also upregulated in response to a range of genotoxic and oxidative agents as well as during starvation. Moreover, CarD was found to be essential for M. smegmatis and $M$. tuberculosis viability and survival during exposure to oxidative stress, DNA damage and nutrient limitation. These data suggested that CarD could be part of a global response network.

Whole-genome transcriptional profiling in $M$. smegmatis revealed that in the absence of CarD there was a substantial upregulation of the expression of genes encoding ribosomal RNAs and other components of the translation machinery, which indicated that CarD may be involved in the stringent response. Bacteria survive periods of starvation and stress by activating the stringent response, which ensures that transcription of ribosomal proteins and stable RNAs such as rRNAs is downregulated. In most bacteria this process is regulated by the nucleotide alarmones guanosine tetraphosphate and guanosine pentaphosphate (collectively referred to as (p)ppGpp), and in Proteobacteria the transcription factor DnaK suppressor protein A (DksA) is also required. Transcriptional control is achieved through direct interaction with RNA polymerase. The involvement of CarD in the stringent response was confirmed by the findings that the absence of CarD enhanced the accumulation of rRNA during nutrient deprivation, oxidative stress and genotoxic stress and that CarD was required for effective control of rRNA transcription by (p)ppGpp. The authors went on to demonstrate that although CarD is a functional homologue of DksA, it interacts with a different site on RNA polymerase. Finally, analysis of the requirement for CarD in a mouse model of infection showed that CarD is required for mycobacterial replication and persistence.

This study identifies CarD as an essential component of the stringent response in mycobacteria and underlines the importance of the stringent response for mycobacterial persistence.

$$
\text { Sheilagh Molloy }
$$

ORIGINAL RESEARCH PAPER Stallings, C. L. et al. CarD is an essential regulator of rRNA transcription required for Mycobacterium tuberculosis persistence. Cell 138, 146-159 (2009) 